Aharonian, F.;...; Rowell, Gavin Peter; ... et al.; H.E.S.S. Collaboration

Limits on an energy dependence of the speed of light from a flare of the active galaxy PKS 2155-304 Physical Review Letters, 2008; 101(17):170402

(C)2008 American Physical Society

http://link.aps.org/doi/10.1103/PhysRevLett.101.170402

\title{
PERMISSIONS
}

http://publish.aps.org/authors/transfer-of-copyright-agreement

"The author(s), and in the case of a Work Made For Hire, as defined in the U.S.

Copyright Act, 17 U.S.C.

$\S 101$, the employer named [below], shall have the following rights (the "Author Rights"):

$[\ldots]$

3. The right to use all or part of the Article, including the APS-prepared version without revision or modification, on the author(s)' web home page or employer's website and to make copies of all or part of the Article, including the APS-prepared version without revision or modification, for the author(s)' and/or the employer's use for educational or research purposes."

$10^{\text {th }}$ May 2013 


\section{Limits on an Energy Dependence of the Speed of Light from a Flare of the Active Galaxy PKS 2155-304}

F. Aharonian, ${ }^{1,13}$ A. G. Akhperjanian, ${ }^{2}$ U. Barres de Almeida, ${ }^{8}$ A. R. Bazer-Bachi, ${ }^{3}$ Y. Becherini, ${ }^{12}$ B. Behera, ${ }^{14}$ M. Beilicke, ${ }^{4}$ W. Benbow, ${ }^{1}$ K. Bernlöhr, ${ }^{1,5}$ C. Boisson, ${ }^{6}$ A. Bochow, ${ }^{1}$ V. Borrel,${ }^{3}$ I. Braun, ${ }^{1}$ E. Brion, ${ }^{7}$ J. Brucker,${ }^{16}$ P. Brun, ${ }^{7}$ R. Bühler, ${ }^{1, *}$ T. Bulik, ${ }^{24}$ I. Büsching, ${ }^{9}$ T. Boutelier,${ }^{17}$ S. Carrigan, ${ }^{1}$ P. M. Chadwick, ${ }^{8}$ A. Charbonnier, ${ }^{19}$ R. C. G. Chaves, ${ }^{1}$ L.-M. Chounet,${ }^{10}$ A. C. Clapson, ${ }^{1}$ G. Coignet, ${ }^{11}$ L. Costamante,${ }^{1,29}$ M. Dalton, ${ }^{5}$ B. Degrange,${ }^{10}$ C. Deill, ${ }^{1}$ H. J. Dickinson, ${ }^{8}$ A. Djannati-Ataï, ${ }^{12}$ W. Domainko, ${ }^{1}$ L. O'C. Drury, ${ }^{13}$ F. Dubois,${ }^{11}$ G. Dubus, ${ }^{17}$ J. Dyks,${ }^{24}$ K. Egberts, ${ }^{1}$ D. Emmanoulopoulos, ${ }^{14}$ P. Espigat, ${ }^{12}$ C. Farnier ${ }^{15}$ F. Feinstein, ${ }^{15}$ A. Fiasson, ${ }^{15}$ A. Förster, ${ }^{1}$ G. Fontaine,${ }^{10}$ M. Füßling, ${ }^{5}$ S. Gabici, ${ }^{13}$ Y. A. Gallant, ${ }^{15}$ L. Gérard, ${ }^{12}$ B. Giebels, ${ }^{10}$ J. F. Glicenstein, ${ }^{7}$ B. Glück, ${ }^{16}$ P. Goret, ${ }^{7}$ C. Hadjichristidis,${ }^{8}$ D. Hauser, ${ }^{14}$ M. Hauser, ${ }^{14}$ S. Heinz, ${ }^{16}$ G. Heinzelmann, ${ }^{4}$ G. Henri, ${ }^{17}$ G. Hermann, ${ }^{1}$ J. A. Hinton, ${ }^{25}$ A. Hoffmann, ${ }^{18}$ W. Hofmann, ${ }^{1}$ M. Holleran, ${ }^{9}$ S. Hoppe,${ }^{1}$ D. Horns,${ }^{4}$ A. Jacholkowska, ${ }^{19,+}$ O. C. de Jager, ${ }^{9}$ I. Jung, ${ }^{16}$ K. Katarzyński, ${ }^{27}$ S. Kaufmann, ${ }^{14}$ E. Kendziorra, ${ }^{18}$ M. Kerschhaggl, ${ }^{5}$ D. Khangulyan, ${ }^{1}$ B. Khélifi, ${ }^{10}$ D. Keogh, ${ }^{8}$ Nu. Komin,${ }^{15}$ K. Kosack, ${ }^{1}$ G. Lamanna, ${ }^{11}$ J.-P. Lenain, ${ }^{6}$ T. Lohse,${ }^{5}$ V. Marandon, ${ }^{12}$ J. M. Martin, ${ }^{6}$ O. Martineau-Huynh, ${ }^{19}$ A. Marcowith,${ }^{15}$ D. Maurin, ${ }^{19}$ T. J. L. McComb,${ }^{8}$ C. Medina, ${ }^{6}$ R. Moderski,${ }^{24}$ E. Moulin,${ }^{7}$ M. Naumann-Godo, ${ }^{10}$ M. de Naurois, ${ }^{19}$ D. Nedbal,${ }^{20}$ D. Nekrassov, ${ }^{1}$ J. Niemiec, ${ }^{28}$ S. J. Nolan, ${ }^{8}$ S. Ohm, ${ }^{1}$ J.-F. Olive, ${ }^{3}$ E. de Oña Wilhelmi, ${ }^{12,29}$ K. J. Orford, ${ }^{8}$ J. L. Osborne, ${ }^{8}$ M. Ostrowski, ${ }^{23}$ M. Panter, ${ }^{1}$ G. Pedaletti, ${ }^{14}$ G. Pelletier, ${ }^{17}$ P.-O. Petrucci, ${ }^{17}$ S. Pita, ${ }^{12}$ G. Pühlhofer, ${ }^{14}$ M. Punch, ${ }^{12}$ A. Quirrenbach,${ }^{14}$ B. C. Raubenheimer, ${ }^{9}$ M. Raue ${ }^{1,29}$ S. M. Rayner, ${ }^{8}$ M. Renaud, ${ }^{1}$ F. Rieger, ${ }^{1,29}$ J. Ripken, ${ }^{4}$ L. Rob, ${ }^{20}$ S. Rosier-Lees,${ }^{11}$ G. Rowell,${ }^{26}$ B. Rudak,${ }^{24}$ J. Ruppel, ${ }^{21}$ V. Sahakian, ${ }^{2}$ A. Santangelo, ${ }^{18}$ R. Schlickeiser,${ }^{21}$ F. M. Schöck, ${ }^{16}$ R. Schröder, ${ }^{21}$ U. Schwanke, ${ }^{5}$ S. Schwarzburg, ${ }^{18}$ S. Schwemmer, ${ }^{14}$ A. Shalchi, ${ }^{21}$ J. L. Skilton, ${ }^{25}$ H. Sol, ${ }^{6}$ D. Spangler, ${ }^{8}$ Ł. Stawarz,${ }^{23}$ R. Steenkamp, ${ }^{22}$ C. Stegmann, ${ }^{16}$ G. Superina, ${ }^{10}$ P. H. Tam, ${ }^{14}$ J.-P. Tavernet, ${ }^{19}$ R. Terrier, ${ }^{12}$ O. Tibolla, ${ }^{14}$ C. van Eldik, ${ }^{1}$ G. Vasileiadis,${ }^{15}$ C. Venter, ${ }^{9}$ J. P. Vialle, ${ }^{11}$ P. Vincent, ${ }^{19}$ M. Vivier, ${ }^{7}$ H. J. Völk, ${ }^{1}$ F. Volpe ${ }^{10,29}$ S. J. Wagner, ${ }^{14}$ M. Ward, ${ }^{8}$ A. A. Zdziarski, ${ }^{24}$ and A. Zech ${ }^{6}$

\section{(H.E.S.S. Collaboration)}

\footnotetext{
${ }^{1}$ Max-Planck-Institut für Kernphysik, P.O. Box 103980, D 69029 Heidelberg, Germany

${ }^{2}$ Yerevan Physics Institute, 2 Alikhanian Brothers Street, 375036 Yerevan, Armenia

${ }^{3}$ Centre d'Etude Spatiale des Rayonnements, CNRS/UPS, 9 av. du Colonel Roche, BP 4346, F-31029 Toulouse Cedex 4, France

${ }^{4}$ Universität Hamburg, Institut für Experimentalphysik, Luruper Chaussee 149, D 22761 Hamburg, Germany

${ }^{5}$ Institut für Physik, Humboldt-Universität zu Berlin, Newtonstr. 15, D 12489 Berlin, Germany

${ }^{6}$ LUTH, Observatoire de Paris, CNRS, Université Paris Diderot, 5 Place Jules Janssen, 92190 Meudon, France

${ }^{7}$ IRFU/DSM/CEA, CE Saclay, F-91191 Gif-sur-Yvette, Cedex, France

${ }^{8}$ University of Durham, Department of Physics, South Road, Durham DH1 3LE, United Kingdom

${ }^{9}$ Unit for Space Physics, North-West University, Potchefstroom 2520, South Africa

${ }^{10}$ Laboratoire Leprince-Ringuet, Ecole Polytechnique, CNRS/IN2P3, F-91128 Palaiseau, France

${ }^{11}$ Laboratoire d'Annecy-le-Vieux de Physique des Particules, CNRS/IN2P3,

9 Chemin de Bellevue - BP 110 F-74941 Annecy-le-Vieux Cedex, France

${ }^{12}$ Astroparticule et Cosmologie (APC), CNRS, Universite Paris 7 Denis Diderot, 10, rue Alice Domon et Leonie Duquet, F-75205 Paris Cedex 13, France

${ }^{13}$ Dublin Institute for Advanced Studies, 5 Merrion Square, Dublin 2, Ireland

${ }^{14}$ Landessternwarte, Universität Heidelberg, Königstuhl, D 69117 Heidelberg, Germany

${ }^{15}$ Laboratoire de Physique Théorique et Astroparticules, CNRS/IN2P3, Université Montpellier II, CC 70, Place Eugène Bataillon, F-34095 Montpellier Cedex 5, France

${ }^{16}$ Universität Erlangen-Nürnberg, Physikalisches Institut, Erwin-Rommel-Str. 1, D 91058 Erlangen, Germany

${ }^{17}$ Laboratoire d'Astrophysique de Grenoble, INSU/CNRS, Université Joseph Fourier, BP 53, F-38041 Grenoble Cedex 9, France

${ }^{18}$ Institut für Astronomie und Astrophysik, Universität Tübingen, Sand 1, D 72076 Tübingen, Germany

${ }^{19}$ LPNHE, Université Pierre et Marie Curie Paris 6, Université Denis Diderot Paris 7, CNRS/IN2P3, 4 Place Jussieu, F-75252, Paris Cedex 5, France

${ }^{20}$ Institute of Particle and Nuclear Physics, Charles University, V Holesovickach 2, 18000 Prague 8, Czech Republic

${ }^{21}$ Institut für Theoretische Physik, Lehrstuhl IV: Weltraum und Astrophysik, Ruhr-Universität Bochum, D 44780 Bochum, Germany

${ }^{22}$ University of Namibia, Private Bag 13301, Windhoek, Namibia

${ }^{23}$ Obserwatorium Astronomiczne, Uniwersytet Jagielloński, ul. Orla 171, 30-244 Kraków, Poland

${ }^{24}$ Nicolaus Copernicus Astronomical Center, ul. Bartycka 18, 00-716 Warsaw, Poland
} 


\title{
${ }^{25}$ School of Physics \& Astronomy, University of Leeds, Leeds LS2 9JT, United Kingdom \\ ${ }^{26}$ School of Chemistry \& Physics, University of Adelaide, Adelaide 5005, Australia \\ ${ }^{27}$ Toruń Centre for Astronomy, Nicolaus Copernicus University, ul. Gagarina 11, 87-100 Toruń, Poland \\ ${ }^{28}$ Instytut Fizyki Jądrowej PAN, ul. Radzikowskiego 152, 31-342 Kraków, Poland \\ ${ }^{29}$ European Associated Laboratory for Gamma-Ray Astronomy, jointly supported by CNRS and MPG
} (Received 10 July 2008; published 22 October 2008)

\begin{abstract}
In the past few decades, several models have predicted an energy dependence of the speed of light in the context of quantum gravity. For cosmological sources such as active galaxies, this minuscule effect can add up to measurable photon-energy dependent time lags. In this Letter a search for such time lags during the High Energy Stereoscopic System observations of the exceptional very high energy flare of the active galaxy PKS 2155-304 on 28 July 2006 is presented. Since no significant time lag is found, lower limits on the energy scale of speed of light modifications are derived.
\end{abstract}

DOI: 10.1103/PhysRevLett.101.170402

Albert Einstein's postulate "that light is always propagated in empty space with a definite velocity $c$ which is independent of the state of motion of the emitting body" [1] is one of the pillars of modern physics. Modification of this postulate would have far-reaching consequences for our understanding of nature; it is, therefore, important to constantly improve the verification of its validity. Particularly in the past few decades, a possible energy dependence of the speed of light has been predicted in the framework of quantum gravity models [2-4] and effective field theory [5], leading to deviations from this postulate (for reviews see [6-8]). The speed of light modifications have different functional dependencies on the photon energy and helicity in different models. Predictions usually entail free parameters such as the relevant mass scale. However, it is commonly expected that this modification should appear at energies of the order of the Planck energy $\left(E_{P}=1.22 \times 10^{19} \mathrm{GeV}\right)$. For energies much smaller than the Planck energy, a series expansion is therefore expected to be applicable, allowing the energy dependence of the speed of light to be parametrized in a model-independent way [6]. The photon speed $c^{\prime}$ is written up to second order in energy $E$ as

$$
c^{\prime}=c\left(1+\xi \frac{E}{E_{P}}+\zeta \frac{E^{2}}{E_{P}^{2}}\right)
$$

where $\xi$ and $\zeta$ are free parameters. Even for the highest photon energies currently measured the corrections are expected to be very small. However, Amelino-Camelia et al. [6] suggested that these minuscule modifications can add up to measurable time delays for photons from cosmological sources. At a redshift $z$, simultaneously emitted photons, with energies $E_{1}$ and $E_{2}$, will arrive at the observer with a time delay $\Delta t=t_{1}-t_{2}$ per energy difference $\Delta E=E_{1}-E_{2}$ of [9]

$$
\frac{\Delta t}{\Delta E} \approx \frac{\xi}{E_{P} H_{0}} \int_{0}^{z} d z^{\prime} \frac{\left(1+z^{\prime}\right)}{\sqrt{\Omega_{m}\left(1+z^{\prime}\right)^{3}+\Omega_{\Lambda}}},
$$

where $\Omega_{m}=0.3, \Omega_{\Lambda}=0.7$ and $H_{0}=70 \mathrm{~km} \mathrm{~s}^{-1} \mathrm{Mpc}^{-1}$ are the cosmological parameters as currently measured. In
PACS numbers: 03.30.+p, 95.85.Pw, 96.50.S-, 98.54.Cm

the case of a vanishing linear term, the mean time delay of the photons per squared energy difference $\Delta E^{2}=E_{1}^{2}-E_{2}^{2}$ is

$$
\frac{\Delta t}{\Delta E^{2}} \approx \frac{3 \zeta}{2 E_{P}^{2} H_{0}} \int_{0}^{z} d z^{\prime} \frac{\left(1+z^{\prime}\right)^{2}}{\sqrt{\Omega_{m}\left(1+z^{\prime}\right)^{3}+\Omega_{\Lambda}}} .
$$

The absence of such an energy dispersion has been used to set bounds on the parameters $\xi$ and $\zeta$. Gamma-ray bursts and very high energies flares of active galaxies have been the primary targets of these "time-of-flight" studies. For the linear dispersion term in Eq. (1), these measurements reach limits of $|\xi|<70-150$ [10-15] for gamma-ray bursts. For active galaxies, dispersion measurements exist for only two sources: Mkn 421 and Mkn 501. Both are located at a similar redshift of $\sim 0.03$. For Mkn 421 , a limit of $|\xi|<200$ was set by the Whipple collaboration during a flare in 1996 [16]. For Mkn 501, an indication of higher energy photons lagging the lower energy ones was reported during a flare in 2005 by the MAGIC collaboration [17]. This dispersion was recently quantified to $|\xi| \sim 30$ [18]. Since the signal is however also marginally consistent with zero dispersion, limits of $|\xi|<60$ and $|\zeta|<2.2 \times 10^{17}$ were derived [18]. While limits in $\xi$ from time-of-flight measurements are approaching unity and probing Planckscale energies, limits on $\zeta$ are generally still far from this domain.

Time-of-flight measurements provide the most direct and model-independent test of the constancy of the speed of light with energy. However, alternative methods set more stringent limits relying on additional assumptions: Limits of $|\xi|<10^{-7}$ are deduced if the speed of light modifications in Eq. (1) are helicity dependent $[19,20]$, as predicted by some of the models [2,5]. Also, constraining limits of $|\xi|<10^{-14}$ and $\zeta>-10^{-6}$ were recently reported in [21] under several assumptions, for example, the sign of the speed of light modification is assumed to be negative or helicity dependent and standard kinematics are required to be valid in a Lorentz-violating regime.

A caveat of time-of-flight measurements is that dispersion might be introduced by intrinsic source effects, which 


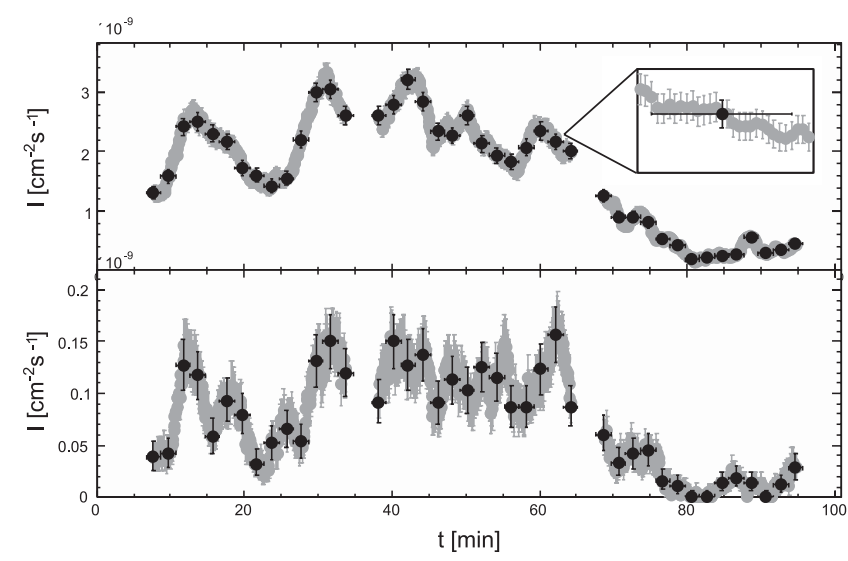

FIG. 1. Black points show the integral flux VHE light curves measured on July 28 from PKS $2155-304$ by H.E.S.S. between 200-800 GeV (upper panel) and $>800 \mathrm{GeV}$ (lower panel), binned in two-minute time intervals. The zero time point is set to MJD 53944.02. Gray points show the oversampled light curve, for which the $2 \mathrm{~min}$. bins are shifted in units of $5 \mathrm{sec}$. The inlay in the upper panel illustrates this in a zoom, where the horizontal error bar shows the duration of the bin in the original light curve.

could cancel out dispersion due to modifications of the speed of light. In the case of a nondetection of dispersion this scenario is unlikely, since it requires both effects to have the same time scale and opposite sign. However, this "conspiracy of nature" [16] can only be ruled out with certainty by observations of sources at multiple distances, as - in contrast to dispersion from speed of light modifications-source intrinsic dispersion should not scale with distance. Population studies of this kind have been performed for gamma-ray bursts, resulting in limits of $|\xi|<$ $1300[8,12-14]$. For active galaxies the data set is currently too sparse to perform these studies.

In the present study, photon time delays were searched for during the VHE flare of the active galaxy PKS 2155-

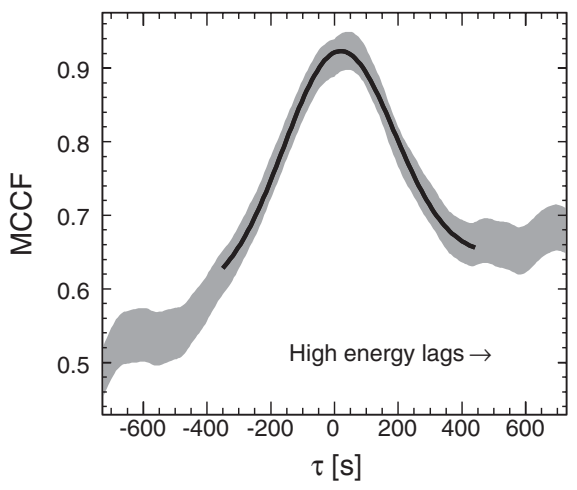

304 observed by the High Energy Stereoscopic System (H.E.S.S.) on July 28 2006. PKS 2155-304 is located at a redshift of $z=0.116$ [22], almost 4 times more distant than Mkn 501 and Mkn 421. The light curve shows fast variability $(\sim 200 \mathrm{~s})$ and covers an energy range of a few $\mathrm{TeV}$ with no significant spectral variability [23]. Considering the unprecedented photon statistics $(\sim 10000$ photons $)$ at these energies, this flare provides a perfect test bed. The data presented here were analyzed using the standard H.E.S.S. analysis, described in detail in [24]. Time delays between light curves of different energies were sought in order to quantify a possible energy dispersion. For this, two different methods were applied, which are described in the following.

The first method determines the time lag between two light curves with the modified cross correlation function (MCCF) [25]. The MCCF is a standard cross correlation function [26], applied to oversampled light curves. This allows time delays below the duration of the flux bins to be resolved [25]. To optimize the energy gap between two energy bands, while keeping good event statistics in both, the correlation analysis was performed on the light curves between 200 and $800 \mathrm{GeV}$ and above $800 \mathrm{GeV}$ (see Fig. 1). The mean difference of the photon energies between the two bands is $1.0 \mathrm{TeV}$ and the mean quadratic difference is 2.0 $\mathrm{TeV}^{2}$. The MCCF of these light curves is shown in Fig. 2. In order to measure the time delay, the central peak of this distribution was fitted by a Gaussian function plus a first-degree polynomial, resulting in a maximum at $\tau_{\text {peak }}=$ $20 \mathrm{~s}$.

The error on the measured time delay is determined by propagating the flux errors via simulations. Ten thousand simulated light curves were generated for each energy band, by varying the flux points of the original oversampled light curve within its measurement errors, taking into account bin correlations. For each pair of light curves, the peak of the MCCF was determined, resulting in a cross

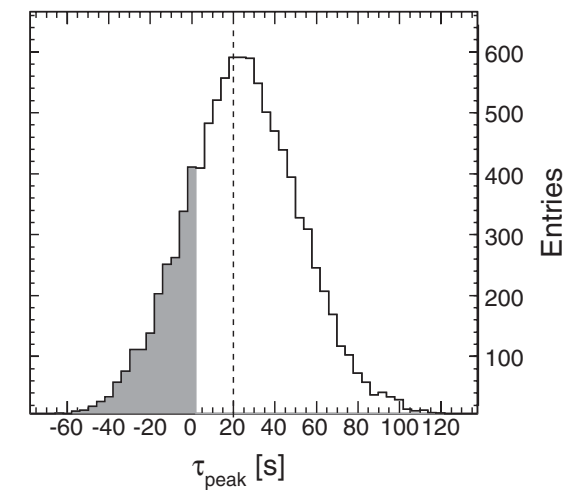

FIG. 2. Left: MCCF of the light curves in Fig. 1. The black line shows the best fit of a Gaussian plus first-degree polynomial. The peak of the fitted function is located at $\tau_{\text {peak }}=20 \mathrm{~s}$. Right: CCPD obtained from 10000 simulated light curves. The shaded area shows the range of the CCPD for $\tau_{\text {peak }} \leq 0$, corresponding to $21 \%$ of the total area. The dotted line shows the position of $\tau_{\text {peak }}$ from the left panel. The CCPD is slightly asymmetric, with a mean of $25 \mathrm{~s}$ and an rms of $28 \mathrm{~s}$ 
correlation peak distribution (CCPD) shown in the right panel of Fig. 2. The CCPD has an rms of $28 \mathrm{~s}$ and yields the probability density of the error of $\tau_{\text {peak }}[27,28]$. For $21 \%$ of the simulations the time delay is negative; therefore, the measured time delay of $20 \mathrm{~s}$ is not significantly different from zero.

The response of the MCCF to energy dispersion is complex. Primarily, dispersion is expected to shift light curves in time according to their mean energy. However, dispersion also broadens their structures and photons might even get shifted out of a burst, decreasing the overall correlation. These "second order" effects become increasingly important once the time shifts approach the time scale of the observed structures in the light curve. The response of the MCCF to dispersion was therefore determined by injecting artificial dispersion into the H.E.S.S. data and measuring its effect on the CCPD. As shown in Fig. 3, the CCPD follows the injected time shift per energy linearly in the range of interest here, confirming the expected behavior. The second order effects mentioned only introduce small deviations, visible at higher dispersion values. Nevertheless, the measured time delays are transformed to dispersion-per-energy with the calibration curve shown in Fig. 3, in order to take these effects into account. Since the measured $\tau_{\text {peak }}$ was compatible with zero, a $95 \%$ confidence upper limit on a linear dispersion of $73 \mathrm{~s} \mathrm{TeV}^{-1}$ is given. Applying the analogous procedure to a quadratic dispersion in energy yields a $95 \%$ confidence limit of $41 \mathrm{~s} \mathrm{TeV}^{-2}$.

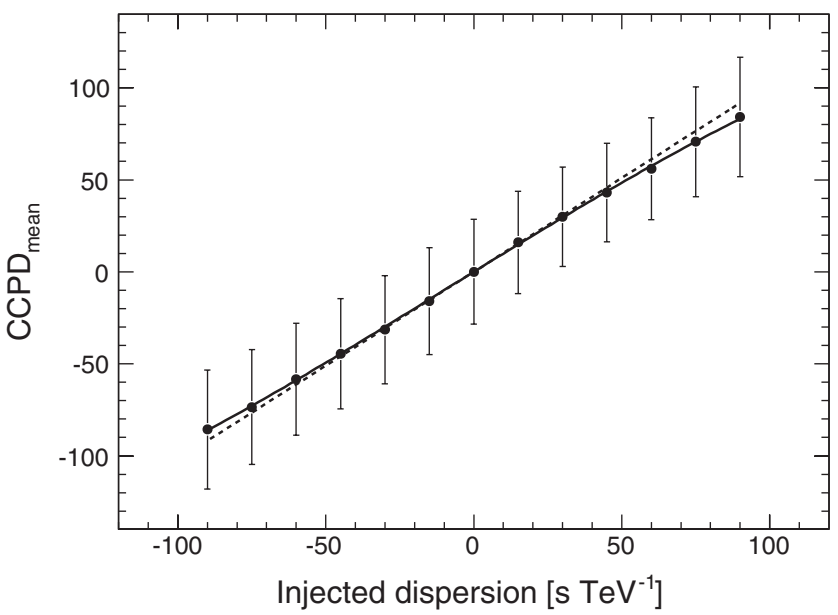

FIG. 3. Mean of the CCPD as a function of the dispersion injected in the H.E.S.S. data. The points have been shifted by the mean value of the CCPD of the original data shown in Fig. 2 to emphasize the relative time shifts. Each CCPD is derived from ten thousand simulated light curves. The error bars show the rms of distributions. The solid line shows the calibration curve used to transform time shifts into dispersion. For comparison, the dotted line shows the linear response function expected from the mean energy of the correlated light curves (see text).
The accuracy of the MCCF method was verified with an independent set of simulations. Eleven thousand new photon lists were generated from the real data using a parametric bootstrap method. The parametric model was obtained from a polynomial spline fit to the light curves in time bins of $1 \mathrm{~min}$ and a fit of the energy distribution of the events in the real data. The CCPD of these new simulations confirmed the previously measured error on the time delay. Artificially introduced dispersion was always recovered within the expected accuracy. It should be noted that the dispersion limit does not depend strongly on the choice of preset parameters, such as the energy ranges and time binning of the light curves and the fit range of the MCCF peak. Varying these parameters within a reasonable range has only a small effect ( $\lesssim 5 \mathrm{~s}$ ) on the final result.

To confirm the result obtained with the MCCF analysis, the dispersion measurement was repeated with an independent method, which is widely applied in time lag studies of GRB light curves [12,13,29]. Light curves were constructed in two energy bands, and a search for extrema was done using a continuous wavelet transform (CWT) [30]. For this the LASTWAVE package [31] was employed, which provides a list of extrema candidates with their positions. The extrema were associated in pairs between light curves and their relative time delay was measured. The association was performed with an algorithm based on the Lipschitz coefficient as in [12,30].

The two energy bands were chosen to be 210 to $250 \mathrm{GeV}$ and above $600 \mathrm{GeV}$, with a mean energy difference of $0.92 \mathrm{TeV}$. Since tiny dispersions are to be probed, a time bin-width of 60 seconds was found to be optimal for this study. The CWT method identified two pairs of extrema with a mean time delay of $27 \mathrm{sec}$. In order to assess the error of this value, samples composed of hundreds of Monte Carlo experiments were analyzed for three linear dispersion values: 0 and $\pm 45 \mathrm{~s} \mathrm{TeV}^{-1}$, in analogy to the MCCF calibration. The values of the error on the measured time lag were found to range between 30 and 36 seconds. The relation between injected dispersion and measured time shift between light curves is again used to derive a limit on the dispersion, resulting in a 95\% confidence limit of $100 \mathrm{~s} \mathrm{TeV}^{-1}$. The impact of systematic effects have also been investigated: selection of gammalike events and the choice of the energy domain or time binning of the light curves change the results by $0.5 \sigma$ at most. Various cuts on the CWT parameters have been applied and lead to negligible changes in the extrema identification.

The measured limits on the energy dispersion translate into limits on the energy scale of speed of light modifications. For a linear dispersion in energy, Eq. (2) yields $|\xi|<$ 17 (or $|\xi|^{-1} E_{p}>7.2 \times 10^{17} \mathrm{GeV}$ ) for the limit obtained with the MCCF method, at $95 \%$ confidence. The linear dispersion limits obtained from the wavelet analysis yields a limit of $|\xi|<23$ (or $|\xi|^{-1} E_{p}>5.2 \times 10^{17} \mathrm{GeV}$ ), confirming this result. These limits are the most constraining 
limits from time-of-flight measurements to date. For a quadratic dispersion in energy, the MCCF method yields $|\zeta|<7.3 \times 10^{19}$ (or $|\zeta|^{-1 / 2} E_{p}>1.4 \times 10^{9} \mathrm{GeV}$ ) with Eq. (3).

This measurement opens a new redshift range for population studies of time delays from active galaxies, which are needed to rule out the possibility of time delay cancellation. For a final verdict on this question further VHE observations of active galaxies are needed. However, the result already shows that the time delay reported for Mkn 501 in [18], if considered significant, cannot be attributed to speed of light modifications. Current and future instruments such as Fermi for gamma-ray bursts, or the proposed Cherenkov Telescope Array for active galaxies, will further improve the sensitivity of time-of-flight measurements, perhaps one day revealing deviations from Einstein's postulate.

The support of the Namibian authorities and of the University of Namibia in facilitating the construction and operation of H.E.S.S. is gratefully acknowledged, as is the support by the German Ministry for Education and Research (BMBF), the Max Planck Society, the French Ministry for Research, the CNRS-IN2P3 and the Astroparticle Interdisciplinary Programme of the CNRS, the U.K. Science and Technology Facilities Council (STFC), the IPNP of the Charles University, the Polish Ministry of Science and Higher Education, the CAPES Foundation, Ministry of Education of Brazil, the South African Department of Science and Technology and National Research Foundation, and by the University of Namibia. We appreciate the excellent work of the technical support staff in Berlin, Durham, Hamburg, Heidelberg, Palaiseau, Paris, Saclay, and in Namibia in the construction and operation of the equipment.

*Rolf.Buehler@mpi-hd.mpg.de

+Agnieszka.Jacholkowska@cern.ch

[1] A. Einstein, Ann. Phys. (Leipzig) 322, 891 (1905); translation from http://www.fourmilab.ch/etexts/einstein/ specrel/www/.

[2] J. Alfaro, H. A. Morales-Técotl, and L.F. Urrutia, Phys. Rev. D 65, 103509 (2002).

[3] J. Ellis, N.E. Mavromatos, and D. V. Nanopoulos, Phys. Lett. B 665, 412 (2008).

[4] G. Amelino-Camelia, G. Mandanici, A. Procaccini, and J. Kowalski-Glikman, Int. J. Mod. Phys. A 20, 6007 (2005).

[5] R. C. Myers and M. Pospelov, Phys. Rev. Lett. 90, 211601 (2003).
[6] G. Amelino-Camelia, J. Ellis, N.E. Mavromatos, D. V. Nanopoulos, and S. Sarkar, Nature (London) 395, 525 (1998).

[7] D. Mattingly, Living Rev. Relativity 8, 5 (2005).

[8] J. Ellis, K. Farakos, N. E. Mavromatos, V. A. Mitsou, and D. V. Nanopoulos, Astrophys. J. 535, 139 (2000).

[9] U. Jacob and T. Piran, J. Cosmol. Astropart. Phys. 01 (2008) 031.

[10] B. E. Schaefer, Phys. Rev. Lett. 82, 4964 (1999).

[11] S. E. Boggs, C. B. Wunderer, K. Hurley, and W. Coburn, Astrophys. J. Lett. 611, L77 (2004).

[12] J. Ellis, N.E. Mavromatos, D. V. Nanopoulos, A. S. Sakharov, and E. K. G. Sarkisyan, Astropart. Phys. 25, 402 (2006).

[13] J. Bolmont, A. Jacholkowska, J.-L. Atteia, F. Piron, and G. Pizzichini, Astrophys. J. 676, 532 (2008).

[14] R. Lamon, N. Produit, and F. Steiner, Gen. Relativ. Gravit. 40, 1731 (2008).

[15] M. Rodríguez Martínez, T. Piran, and Y. Oren, J. Cosmol. Astropart. Phys. 05 (2006) 017.

[16] S. D. Biller et al. (Whipple Collaboration), Phys. Rev. Lett. 83, 2108 (1999).

[17] J. Albert et al. (MAGIC Collaboration), Astrophys. J. 669, 862 (2007).

[18] J. Albert et al. (MAGIC Collaboration), Phys. Lett. B 668, 253 (2008).

[19] R. J. Gleiser and C. N. Kozameh, Phys. Rev. D 64, 083007 (2001).

[20] Y.-Z. Fan, D.-M. Wei, and D. Xu, Mon. Not. R. Astron. Soc. 376, 1857 (2007).

[21] M. Galaverni and G. Sigl, Phys. Rev. Lett. 100, 021102 (2008).

[22] R. Falomo, J. E. Pesce, and A. Treves, Astrophys. J. 411, L63 (1993).

[23] F. Aharonian et al. (H.E.S.S. Collaboration), Astrophys. J. 664, L71 (2007).

[24] F. Aharonian et al. (H.E.S.S. Collaboration), Astron. Astrophys. 457, 899 (2006).

[25] T.-P. Li, J.-L. Qu, H. Feng, L.-M. Song, G.-Q. Ding, and L. Chen, Chin. J. Astron. Astrophys. 4, 583 (2004).

[26] R. A. Edelson and J.H. Krolik, Astrophys. J. 333, 646 (1988).

[27] D. Maoz and H. Netzer, Mon. Not. R. Astron. Soc. 236, 21 (1989).

[28] B. M. Peterson, I. Wanders, K. Horne, S. Collier, T. Alexander, S. Kaspi, and D. Maoz, Publ. Astron. Soc. Pac. 110, 660 (1998).

[29] J. Ellis, N. E. Mavromatos, D. V. Nanopoulos, and A. S. Sakharov, Astron. Astrophys. 402, 409 (2003).

[30] S. Mallat, A Wavelet Tour of Signal Processing (Academic Press, San Diego, 1999).

[31] E. Bacry, LASTWAVE version 2.0.3 (2004), http:// www.cmap.polytechnique.fr/ bacry/LastWave/. 